\title{
Online Energy Management of Plug-In Hybrid Electric Vehicles for Prolongation of All-Electric Range Based on Dynamic Programming
}

\author{
Zeyu Chen, ${ }^{1}$ Weiguo Liu, ${ }^{2}$ Ying Yang, ${ }^{1}$ and Weiqiang Chen ${ }^{1,3}$ \\ ${ }^{1}$ School of Mechanical Engineering and Automation, Northeastern University, Shenyang 110819, China \\ ${ }^{2}$ Zhejiang Key Laboratory of Automobile Safety Technology, Hangzhou 310000, China \\ ${ }^{3}$ BYD Auto Industry Co., Ltd., Shenzhen 518000, China \\ Correspondence should be addressed to Zeyu Chen; chenzy@mail.neu.edu.cn
}

Received 4 September 2015; Revised 21 November 2015; Accepted 29 November 2015

Academic Editor: Xiaosong $\mathrm{Hu}$

Copyright ( 2015 Zeyu Chen et al. This is an open access article distributed under the Creative Commons Attribution License, which permits unrestricted use, distribution, and reproduction in any medium, provided the original work is properly cited.

\begin{abstract}
The employed energy management strategy plays an important role in energy saving performance and exhausted emission reduction of plug-in hybrid electric vehicles (HEVs). An application of dynamic programming for optimization of power allocation is implemented in this paper with certain driving cycle and a limited driving range. Considering the DP algorithm can barely be used in real-time control because of its huge computational task and the dependence on a priori driving cycle, several online useful control rules are established based on the offline optimization results of DP. With the above efforts, an online energy management strategy is proposed finally. The presented energy management strategy concerns the prolongation of all-electric driving range as well as the energy saving performance. A simulation study is deployed to evaluate the control performance of the proposed energy management approach. All-electric range of the plug-in HEV can be prolonged by up to $2.86 \%$ for a certain driving condition. The energy saving performance is relative to the driving distance. The presented energy management strategy brings a little higher energy cost when driving distance is short, but for a long driving distance, it can reduce the energy consumption by up to $5.77 \%$ compared to the traditional CD-CS strategy.
\end{abstract}

\section{Introduction}

Circumstance pollution, energy crisis, and global warming aggravated by urban transportation have attracted remarkable attention recently, impelling the rapid development of alternative energy-based vehicles, among which the HEVs have been recognized as a promising type of vehicle due to their potential to enhance the energy economy and reduce exhausted emission [1]. The advantages of HEVs benefit from two onboard energy sources, gasoline and electricity, which enable the power demand to be split between the engine and onboard battery pack. According to the capacity of onboard battery pack and its capability to be recharged directly from power grid, HEVs can be further categorized into normal HEVs and plug-in HEVs. Unlike the traditional normal HEV s that can only be operated at a charge sustaining (CS) manner, plug-in HEVs can also be operated at charge depleting (CD) manner thanks to the possession of onboard large capacity battery pack [2]. Plug-in HEVs can achieve better energy saving performance compared to normal HEVs, but the operation manners and power allocation become more complicated accordingly due to multiple operation manners.

Energy management strategy plays a significant role in the supervisory control of both normal HEVs and plug-in HEVs to reach the optimal power split policy [3-5]. The energy management problem can be normally formulated as an optimization problem, which is to minimize a cost function by determining the rational operation manner and optimal power allocation. To resolve this problem, several advance optimization algorithms have been employed [6-11], the most representative of which is dynamic programming (DP). For example, Zhang and Xiong [12] deployed DP-based adaptive energy management on different driving conditions, 
incorporating a fuzzy driving condition recognition algorithm for plug-in HEVs. Pérez and Pilotta [13] implemented DP to resolve the energy management problem of HEVs on the finite time horizon and used the optimization results as a benchmark for other strategies' design and components sizing optimization. The implementation of DP relies on an a priori driving cycle. According to the driving cycle it uses, specific or random, DP algorithm can be further divided into two categories: deterministic DP and stochastic DP.

Although the deterministic DP is effective in reaching the global optimality, it can barely be used in real-time control because the specific driving cycle is unknown. A viable method for the deterministic DP to be utilized in the online application is to operate DP offline with some representative driving cycles first and then extract online control policy from the optimization results [14]. The drawback of this method is that the extraction process may lose optimality to some extent. But control performance of the control policy extracted from offline optimization has been recognized as better than that of intuition-based control algorithm [15]. Unlike deterministic DP, stochastic DP is to implement DP algorithm based on prediction of future driving condition, which is obtained by stochastic method using a discrete-time Markov chain $[16,17]$. The potential of stochastic DP if useful information of driving cycle is available has been investigated in [18] by dividing the prediction into three levels. Stochastic DP can achieve a near-optimal control performance, and it is time-invariant and capable of being implemented online. Stochastic DP does not rely on a specific known driving cycle as requisite to implement, but it is sensitive to the real-world driving condition as well because the transition probabilities in Markov chain are based on certain collected driving cycles [19]; if the actual driving condition is obviously different from the collected driving data, stochastic DP algorithm cannot always guarantee the validity.

The method that extracts online control policy based on offline DP optimization is employed in this paper. Three contributions have been made. (1) The deterministic DP algorithm is implemented based on certain driving cycle and a limited driving distance. The presented DP algorithm-based optimal strategy is to determine the optimal control policy of power allocation between engine and battery pack in the CS operation manner. (2) An online energy management approach is proposed for the uncertain real-time control application with consideration of the prolongation of allelectric driving range. In this approach, the $\mathrm{CD}$ manner and CS manner are switched not only by battery state of charge (SoC) but also by the power request level at each time step. Power allocation in CS manner is determined by the control policy extracted from DP to guarantee the nearoptimal energy saving performance. (3) The performance of the presented energy management approach is evaluated by a simulation study and compared to the traditional CD-CS strategy. The correlation between energy saving performance and driving distance is preliminarily disclosed.

The remainder of this paper is organized as follows: configuration of the studied plug-in HEV is introduced and modeled in Section 2; the formulation of energy management problem and dynamic programming algorithm and its

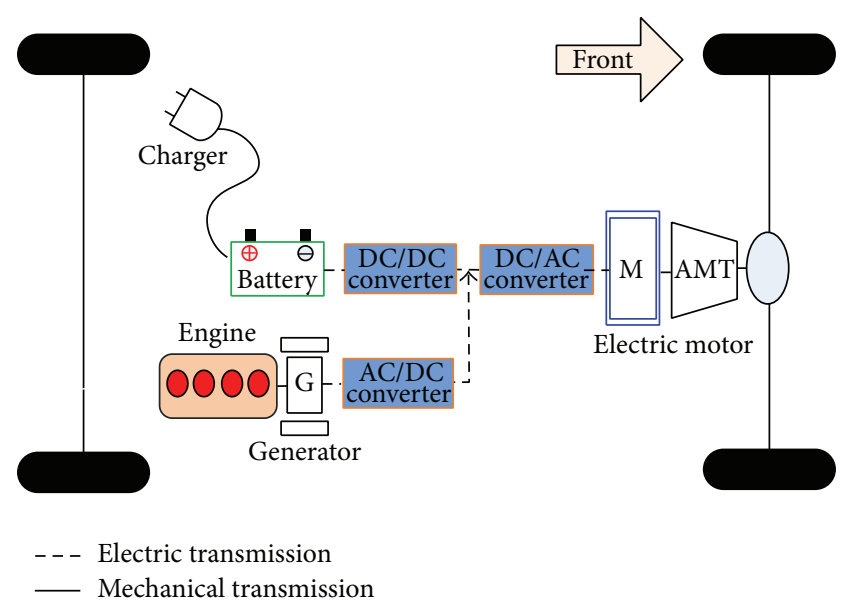

FIGURE 1: Configuration of the plug-in HEV power system.

implementation are described in Section 3. The online energy management strategy against uncertain driving condition is proposed in Section 4. Simulation results and analysis are shown in Section 5 while the conclusions are listed in Section 6.

\section{System Model}

Configuration of the powertrain in the studied plug-in HEV is shown in Figure 1. An electric motor is coupled through an automatic mechanical transmission (AMT) on the fist axle. A lithium-ion battery pack is used as energy storage system (ESS) while the engine-generator set is used as auxiliary power unit (APU). Parameters of the main components are listed in Table 1.

Dynamics effects of the powertrain components are neglected because they are much faster than energy consumption variation and do not affect the power flow distribution [20]; thus, the power balance relationship is modeled as

$$
P_{\mathrm{ESS}}(t)+P_{\mathrm{APU}}(t)=P_{\text {req }}(t),
$$

where $P_{\mathrm{ESS}}, P_{\mathrm{APU}}$ are output power from ESS and APU, respectively, and $P_{\text {req }}$ is defined as the power request of driving electric motor, calculated as follows:

$$
\begin{aligned}
P_{\mathrm{ESS}}(t) & = \begin{cases}P_{B}(t) \eta_{\text {batt }}, & P_{B} \geq 0 \\
\frac{P_{B}(t)}{\eta_{\mathrm{batt}}}, & P_{B}<0,\end{cases} \\
P_{\mathrm{APU}}(t) & =P_{E}(t) \eta_{A}, \\
P_{\mathrm{req}}(t) & = \begin{cases}\frac{T_{m}(t) \omega_{m}(t)}{\eta_{\text {mot }}}, & T_{m}(t) \geq 0 \\
T_{m}(t) \omega_{m}(t) \eta_{\mathrm{mot}}, & T_{m}(t)<0,\end{cases}
\end{aligned}
$$

where $P_{B}(t)$ is battery power, positive value represents discharging, negative value represents charging, $P_{E}(t)$ is engine power, $T_{m}$ is the torque of electric motor, positive value 
TABLE 1: Parameters of main components.

\begin{tabular}{lcc}
\hline Components & Parameters & Value \\
\hline \multirow{2}{*}{ Engine } & Peak power $(\mathrm{kW})$ & 75 \\
& Optimal point $\left(\mathrm{kW} @ \mathrm{r} \cdot \mathrm{min}^{-1}\right)$ & $30 @ 2200$ \\
\hline \multirow{3}{*}{ Electric motor } & Peak power $(\mathrm{kW})$ & 155 \\
& Maximum torque $(\mathrm{Nm})$ & 780 \\
& Maximum speed $(\mathrm{r} / \mathrm{min})$ & 5500 \\
\hline \multirow{2}{*}{ Battery } & Capacity $(\mathrm{Ah})$ & 85 \\
& Rated voltage $(\mathrm{V})$ & 380 \\
\hline
\end{tabular}

represents driving, negative value represents braking, $\omega_{m}$ is the rotate speed of electric motor, and $\eta_{\text {batt }}(t), \eta_{A}(t)$, and $\eta_{\text {mot }}(t)$ are the efficiency of battery pack, APU, and electric motor, respectively.

2.1. Battery Model. Battery pack is modeled by an equivalent circuit analogy. The impact of temperature variation is neglected in this study; then the battery terminal voltage is

$$
V_{\text {batt }}(t)=V_{\text {oc }}(\operatorname{SoC}(t))-R_{0}(\operatorname{SoC}(t)) \cdot I_{\text {batt }}(t),
$$

where $V_{\text {oc }}$ and $R_{0}$ are the open circuit voltage and internal resistance.

The Ampere-hour counting approach is adopted to calculate SoC:

$$
\operatorname{SoC}(t)=\operatorname{SoC}\left(t_{0}\right)-\frac{1}{Q_{\text {nom }}} \int_{t_{0}}^{t} I_{\text {batt }}(t) d t,
$$

where $Q_{\text {nom }}$ is the nominal capacity of battery.

2.2. Engine-Generator Model. The electric generator driven through a speed-increase gearbox by the engine is connected with the DC bus by an uncontrolled rectifier. The equivalent electric circuit diagram of electric generator and rectifier is shown in Figure 2, while Figure 3 gives the efficiency map of the engine. Based on the equivalent circuit, the relationship among the engine-generator output current, the DC bus voltage, the torque, and the speed of the electric generator can be obtained as

$$
\begin{aligned}
& \omega_{g}(t)=\frac{U_{\mathrm{dc}}(t)}{K_{e}-I_{A}(t) K_{x}}, \\
& T_{g}(t)=K_{e} I_{A}(t)-K_{x} I_{A}(t)^{2},
\end{aligned}
$$

where $T_{g}(t)$ and $\omega_{g}(t)$ are the output torque and speed of the electric generator, respectively, $I_{\mathrm{A}}(t)$ is the current of DC bus, $K_{e}$ is the equivalent electromotive force coefficient, $K_{x}$ is the equivalent resistance coefficient, and $U_{\mathrm{dc}}(t)$ is the DC bus voltage.

According to the electric generator torque and the target engine speed, the engine torque is dynamically adjusted by the engine controller. The relationship between the engine torque $T_{e}$ and the electric generator torque $T_{g}$ is

$$
T_{e}(t)=\frac{1}{i_{z} \eta_{z}}\left(T_{g}-\left(\frac{J_{e}}{i_{z}^{2}}+J_{g}\right) \frac{d \omega_{g}(t)}{d t}\right),
$$

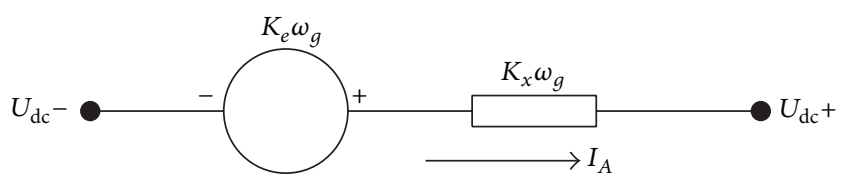

FIGURE 2: Equivalent circuit of generator and rectifier.

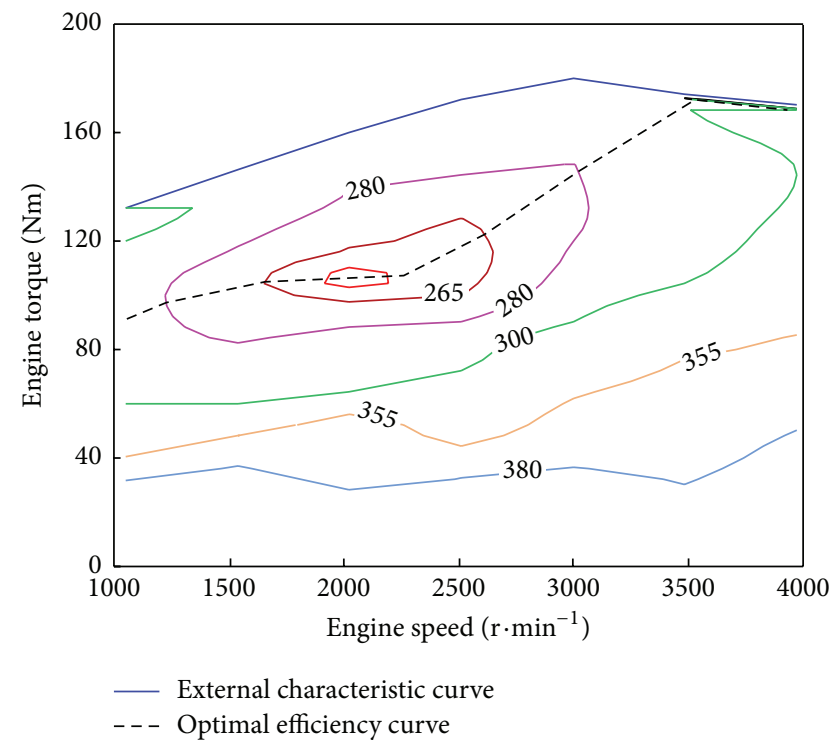

FIGURE 3: The universal characteristics curve of engine.

where $J_{e}$ is engine rotational inertia, $J_{g}$ is the electric generator rotational inertia, and $i_{z}$ and $\eta_{z}$ are the speed-increase gearbox transmission ratio and mechanical efficiency.

2.3. Electric Motor Model. The electric motor is modeled by using the steady-state experimental data with a dynamic correction. Figure 4 gives the external characteristic and efficiency map of the adopted electric motor. The torque of electric motor is determined by the driver operation, as shown in (7), in which the dynamic response process is equivalent to a first-order delay:

$$
T_{m}(t)= \begin{cases}\frac{1}{1+\tau s} T_{m \_ \text {max }}\left(\omega_{m}(t)\right)\left(\lambda_{d}^{*}-\varepsilon u\right), & \lambda_{d}^{*} \geq 0 \\ \frac{1}{1+\tau s} T_{m_{-} \min }\left(\omega_{m}(t)\right) \lambda_{d}^{*}, & \lambda_{d}^{*}<0\end{cases}
$$

where $\tau$ is the time constant of torque dynamic response, $\lambda_{d}^{*}$ is the operation signal of driver pedals, positive value represents driving pedal, negative value represents braking pedal, $T_{m \text { max }}$ and $T_{m \_ \text {min }}$ are the upper and lower limit of motor torque under the current motor speed, $u$ is the vehicle velocity, and $\varepsilon$ is a small positive constant, used as a velocity feedback gain to improve the control stability.

2.4. Vehicle Model. Since the electric motor is mechanically connected to the wheel, the motor rotate speed $\omega_{m}(t)$ can be 


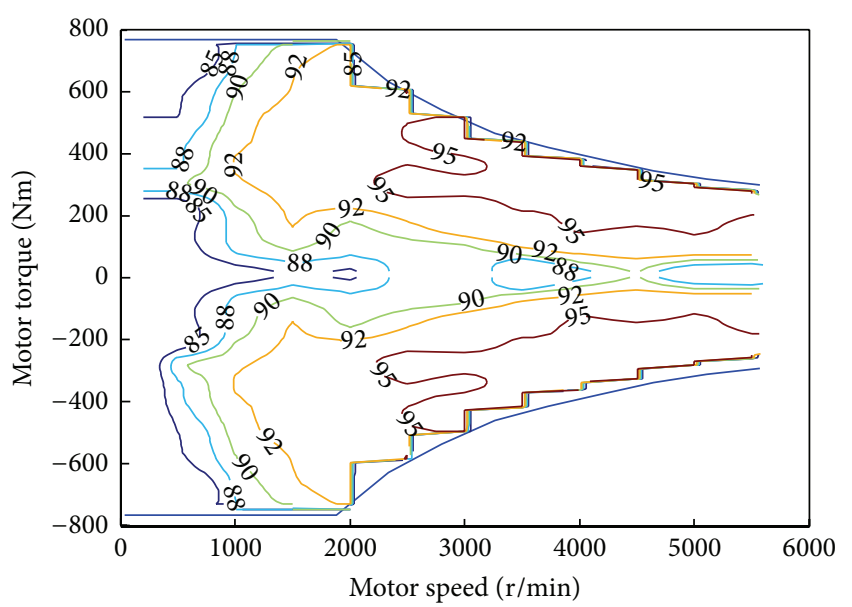

FIGURE 4: Efficiency map of electric motor.

calculated from the vehicle speed. For the energy management problem, the yawing force is neglected, and then the longitudinal dynamics is

$$
\begin{aligned}
& u(t) \\
& =\frac{1}{\delta m} \int_{t_{0}}^{t}\left(\frac{T_{m}(t) i_{T} \eta_{\mathrm{mot}}}{r_{w}}-m g \psi-\frac{1}{2} C_{d} \rho_{a} A u(t)^{2}\right) d t,
\end{aligned}
$$

where $m$ is vehicle mass, $\delta$ is correction coefficient of rotating mass, $g$ is gravitational acceleration, $C_{d}, \rho_{a}$, and $A$ are coefficient of air resistance, air density, and vehicle frontal area, respectively, $\psi$ is coefficient of road resistance, $i_{T}$ is transmission ratio, and $r_{w}$ is the radius of wheel.

The values of coefficients in models are shown in Table 2.

\section{Optimization Algorithm}

3.1. Formulation of Optimal Energy Management Problem. The purpose of DP algorithm here is to obtain the optimal power allocation between battery pack and engine in CS manner. As mentioned above, plug-in HEVs can be operated both in CD manner and in CS manner. The engine turns off in CD manner and battery pack is used to supply power to electric motor single-handedly so as to achieve a clean and economically driving manner. Obviously, optimization process does not work at this situation because only electricity is consumed. When CS manner is adopted, power demand is split between engine and battery; this is where DP algorithm comes in to play; the optimization objective is denoted as the summation of energy cost from two energy sources:

$$
J(u)=m_{\mathrm{equ}}=\int_{t_{0}}^{t_{f}}\left(\dot{m}_{f}(t)+\dot{m}_{\mathrm{batt}}(t)\right) d t
$$

where $m_{f}$ and $m_{\text {batt }}$ are the fuel and electricity consumption, respectively, $t_{0}$ represents the time moment that the vehicle switches to CS manner, and $t_{f}$ is the terminal time of CS manner.
TABLE 2: The values of coefficients in models.

\begin{tabular}{lc}
\hline The coefficients & Value or range \\
\hline$\lambda_{d}$ & {$[-1,1]$} \\
$\varepsilon$ & 0.002 \\
$i_{z}$ & 2 \\
$K_{e}$ & 1.65 \\
$K_{x}$ & 0.00037 \\
$\tau$ & 0.46 \\
$C_{d}$ & 0.3 \\
$A\left(\mathrm{~m}^{2}\right)$ & 2.2 \\
$\psi$ & 0.018 \\
\hline
\end{tabular}

Considering the battery will be unsafe when SoC is quite low, a penalty function is introduced into the "cost function," described as

$$
J(u)=m_{\text {equ }}=\int_{t_{0}}^{t_{f}}\left(\dot{m}_{f}(t)+\dot{m}_{\text {batt }}(t)\right) d t+\sigma(\text { SoC }),
$$

where $\sigma(\mathrm{SoC})$ is a penalty value when SoC is quite low.

The energy cost rate of oil and electricity can be calculated by

$$
\begin{aligned}
\dot{m}_{f}(t) & =f_{E}\left(\omega_{\text {opt }}\left(P_{E}(t)\right), P_{E}(t)\right) \cdot P_{E}(t), \\
\dot{m}_{\text {batt }}(t) & =Q_{\text {equ }} \cdot P_{B}(t),
\end{aligned}
$$

where $f_{E}()$ is the look-up table function from engine efficiency map, $\omega_{\text {opt }}$ is rotate speed of engine corresponding to optimal efficiency, and $Q_{\text {equ }}$ is the equivalent factor to transform the electric energy to fuel consumption.

The control variable is set as $u=\left[P_{E}, P_{B}\right]^{T}$; thus, the optimal control problem can be described as to find an optimal control policy $u^{*}$ to minimize the cost function:

$$
J\left(u^{*}\right) \leq J(u) \quad \forall u \in U .
$$

To ensure the optimal control policy obtained by DP belongs to feasible solutions, the control variables are subject to some constraints.

3.1.1. Inequality Constraints. Inequality constraints define the power limitations for the operation characteristics of components in power system, described as

$$
\begin{aligned}
0 & \leq P_{E} \leq P_{E \_ \text {max }}, \\
P_{B \_ \text {min }} & \leq P_{B} \leq P_{B \_ \text {max }}, \\
\mathrm{SoC}_{\min } & \leq \mathrm{SoC} \leq \mathrm{SoC}_{\max },
\end{aligned}
$$

where $P_{E \_ \text {max }}$ is upper limit of engine power, $P_{\text {batt_min }}$ and $P_{\text {batt_max }}$ are upper limit and lower limit of battery power, respectively, and $\mathrm{SoC}_{\min }$ and $\mathrm{SoC}_{\max }$ are the upper limit and lower limit of battery SoC, respectively.

3.1.2. Equality Constraints. The engine power and battery power are subject to the equality constraint described in (1). 
The power request and battery $\mathrm{SoC}$ are used to represent system state, denoted as $x=\left[P_{\text {req }}, \mathrm{SoC}\right]^{T}$; the equality constraint of initial system state is

$$
x\left(t_{0}\right)=x_{0},
$$

where $x_{0}$ is the initial system state when CS manner is switched on.

The system state is uncertain at terminal due to the existence of CD manner. Therefore, the equality constraint of terminal should be eliminated for plug-in HEVs. However, in order to implement the DP algorithm, an equality constraint is put on the terminal state, described as

$$
x\left(t_{f}\right)=x_{f},
$$

where $x_{f}$ is the system state at the end of CS manner.

3.2. Dynamic Programming. A deterministic DP algorithm is employed to resolve the optimization problem formulated above. DP is a discrete-time global optimal algorithm based on a property that no matter what the previous decisions constitute, the remaining decisions should be an optimal policy. Firstly the previous control problem is rewritten in a discrete-time form for DP application, as shown in Figure 5. At each time step $k(k=n-1, n-2, \ldots, 0)$, the function $J$ when moving from the time step $k$ to the end of the optimization horizon is calculated.

The optimization principle is applied from the end step; when $k=n$, there is $J_{n}(x)=\varphi\left(x_{n}\right)$. Then for each step,

$$
J_{k}(x)=J_{k+1}(x)+\min _{u_{k} \in U}\left(L\left(x_{k}, u_{k}, t\right)\right) .
$$

Starting from the end step to first step, the total cost can be deduced as

$$
J_{0}(\pi)=\varphi\left(x_{n}\right)+\sum_{0}^{n-1} L\left(x_{k}, u_{k}, t\right),
$$

where $\pi=\left[u_{0}, u_{1}, \ldots, u_{n-1}\right]$ is the discrete control policy.

After the DP operates from the end step to the start point, the optimal control policy $\pi^{*}$ can be determined. Since engine power and battery power are subject to an equality constraint, engine power $P_{E}$ is chosen as the control variable of DP to be discretized at each iterative step, as shown in Figure 3. The terminal state variable is set in advance and then, at each step, $\mathrm{SoC}_{k}$ can be deduced by $\mathrm{SoC}_{k+1}$ with the control policy $P_{E}(k)$ :

$$
\operatorname{SoC}(k)=\operatorname{SoC}(k+1)+\frac{\left(P_{\text {req }}(k)-P_{E}(k)\right) \Delta t}{\eta_{\text {batt }} Q_{\text {nom }}} .
$$

3.3. Implementation of Optimization Algorithm. Two driving cycles (HWFET and IM240, as shown in Figure 6) are used to implement DP algorithm to resolve the previous optimization problem. As the power allocation is quite clear in CD manner, DP is only employed to obtain the optimal power allocation in CS manner, in which the battery and engine output power together meet the power demand.

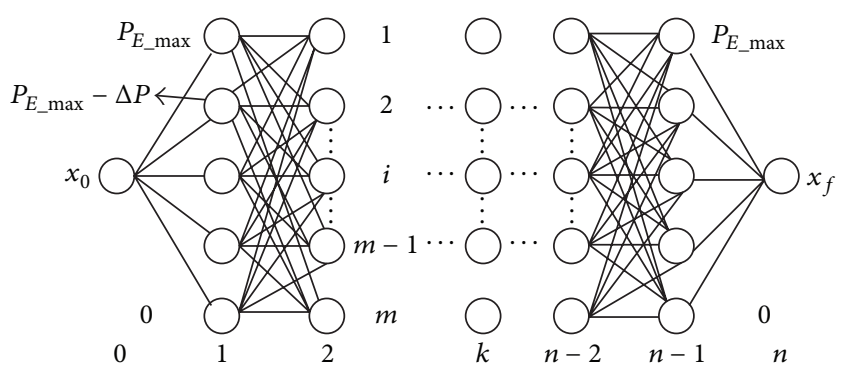

Figure 5: Discrete-time state for DP algorithm.

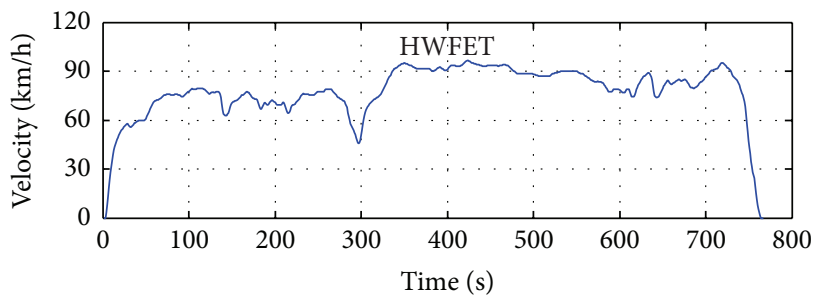

(a)

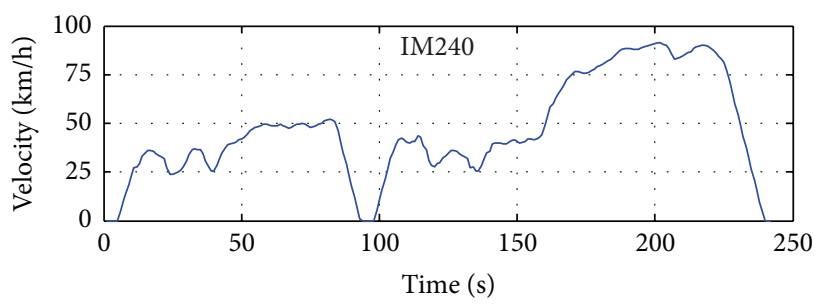

(b)

FIgURE 6: Driving cycles for DP implementation.

Figure 7 shows the optimization results of power allocation in two driving cycles. Although DP cannot be directly used in real-time control, it can provide some useful information about the rational power allocation. Two thresholds $\chi_{1}$ and $\chi_{2}$ are defined as engine power control instructions with the analysis of DP optimization results, described as follows:

(1) It can be concluded that the engine is turned off by DP when the power request from engine is low. Thereby, $\chi_{1}$ is chosen as the threshold to determine the lower limit of the engine output power (here, $\chi_{1}$ is assigned as $30 \mathrm{~kW}$ ).

(2) Similarly, $\chi_{2}$ is defined as a threshold corresponding to the upper limits of engine output power in Figures $7(\mathrm{a})$ and $7(\mathrm{c})$ (here, $\chi_{2}$ is assigned as $42 \mathrm{~kW}$ ). When power request exceeds this threshold, the rest of power demand is provided by the battery.

\section{Online Energy Management Strategy}

Based on the optimization results of power allocation by DP, some specific control rules will be extracted and further form the online energy management strategy in this section. As mentioned above, the operation manner of plug-in HEVs is 


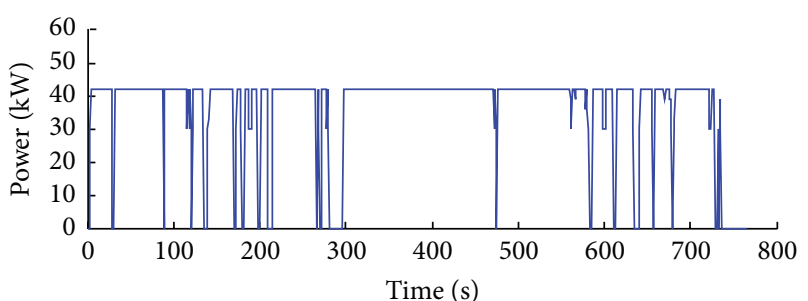

(a)

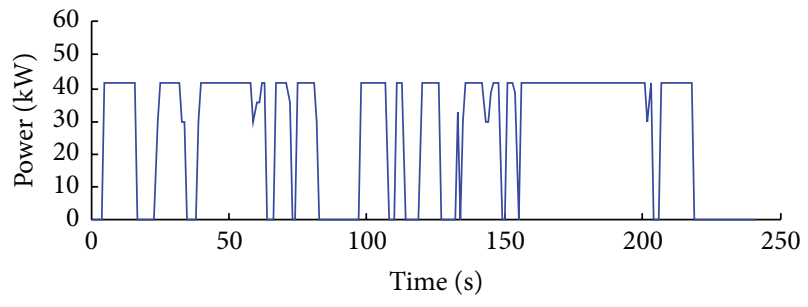

(c)

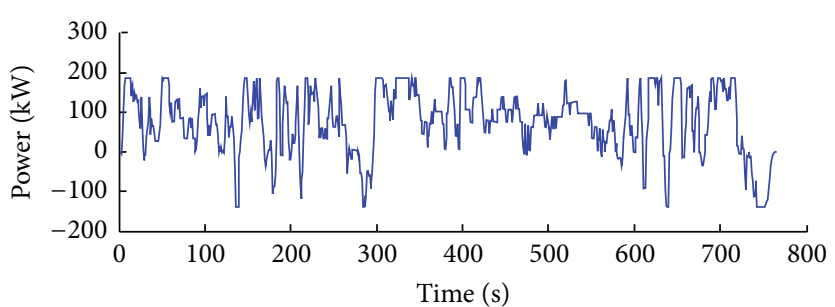

(b)

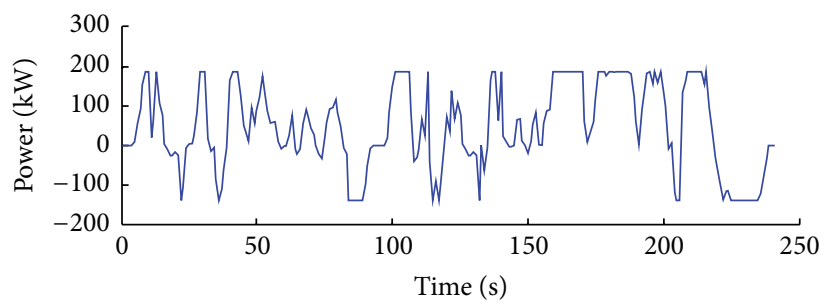

(d)

FIGURE 7: Optimization results of DP algorithm from two driving conditions. (a) Engine power in HWFET. (b) Battery power in HWFET. (c) Engine power in IM240. (d) Battery power in IM240.

divided into $\mathrm{CD}$ and $\mathrm{CS}$ manner. The driving range covered by $\mathrm{CD}$ manner is zero emissions, referred to as all-electric range (AER). In existing research results, the most common used online energy management approach is CD-CS strategy [21], which utilizes the CD manner at first and switches to CS manner when a long driving distance is required; the boundary between CD and CS manner is usually controlled according to the threshold of battery SoC. The shortage of CD-CS strategy is that the control policies are independent with power request of driving condition. For example, if the front part of the route has a quite high power request, according to the CD-CS strategy, vehicle will operate at CD manner at first and the electricity will be consumed at a rapid speed, resulting in a very short AER. In this paper, prolongation of AER is considered as a control objective of the online energy management.

The presented energy management strategy contains two layers: the top layer is to determine the operation manner and the bottom layer concerns the specific power allocation based on DP. Figure 8 shows operation manner control policy in the top layer. Unlike the traditional CD-CS strategy that uses a clear boundary to divide the entire trip into CD and CS stages, here CD manner and CS manner are fused together with each other during vehicle utilization. Besides battery SoC, the power request level at each time is a reference of operation manner control as well. The specific control rules in the top layer can be further described as follows:

Critical Condition 1: High Power Request. When $P_{\text {req }}$ exceeds a threshold $P_{M}$, no matter how high the battery SoC is, the vehicle operation is switched to CS manner, denoted as $\mathrm{CS}^{(P)}$, representing the fact that this CS manner is triggered by power request. The engine output power, according to DP, electricity will continue being consumed in this manner due to the high $P_{\text {req }}$, but the rate of electricity consumption is reduced.

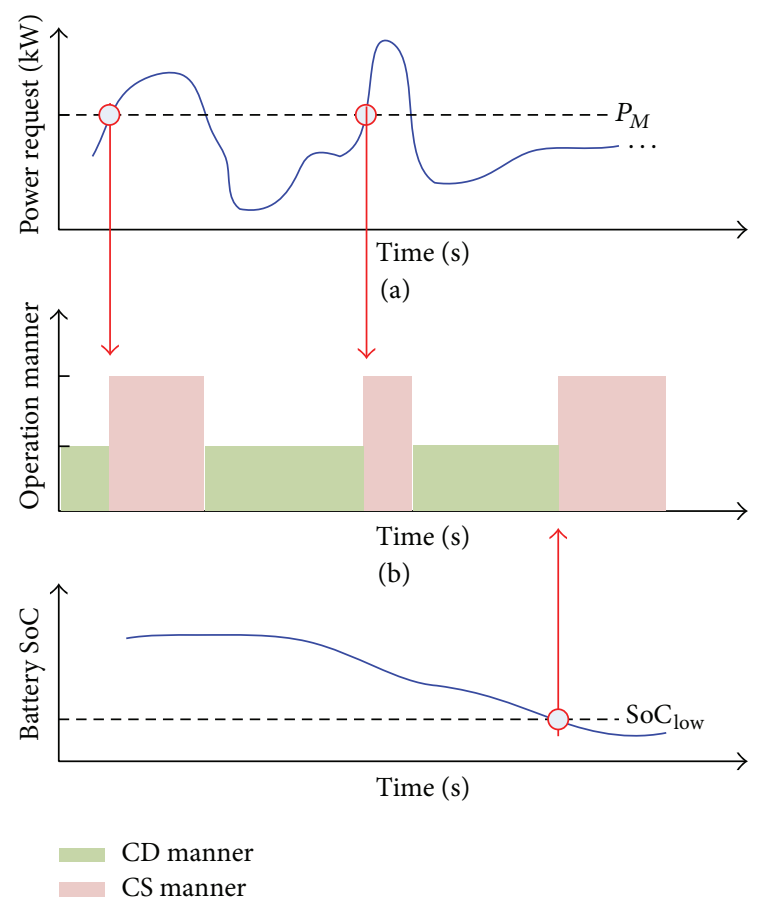

(c)

FIGURE 8: Schematic diagram of online operation manner control strategy. (a) Power request. (b) Operation manner: CD or CS. (c) Battery SoC.

Critical Condition 2: Low SoC. CS manner is adopted when $\mathrm{SoC}$ reaches the threshold $\mathrm{SoC}_{\mathrm{Low}}$, denoted as $\mathrm{CS}^{(B)}$, representing the fact that this CS manner is triggered by battery SoC. Different from $\mathrm{CS}^{(P)}$, in this manner, electricity is not utilized to supply the power request any longer. 


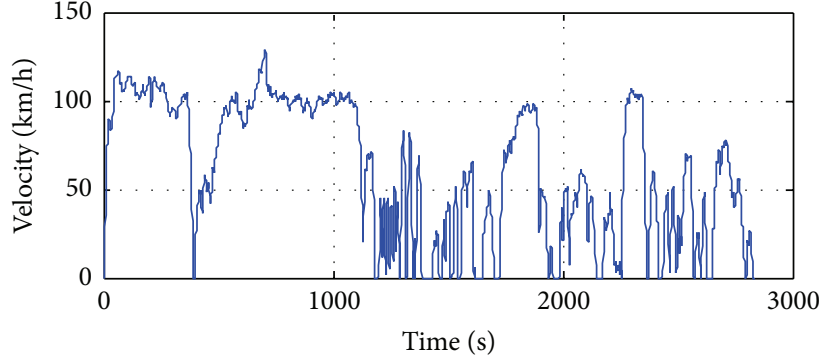

(a)

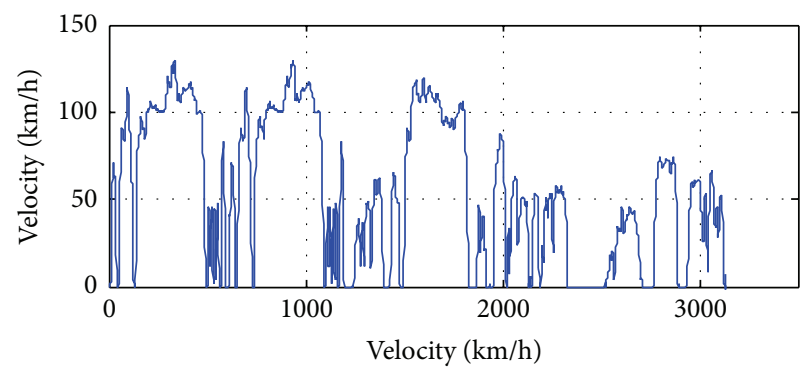

(c)

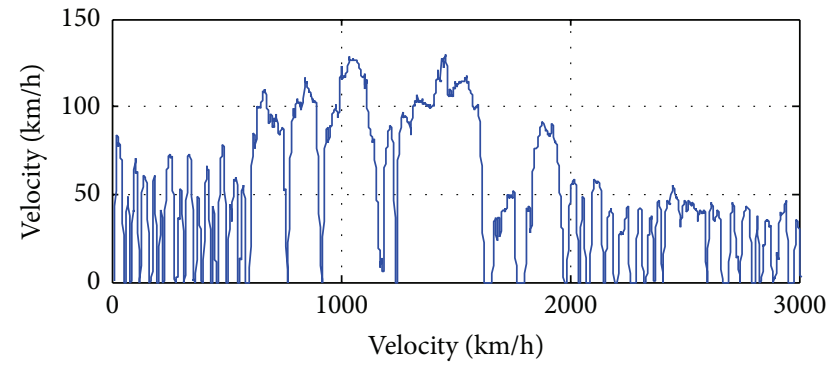

(e)

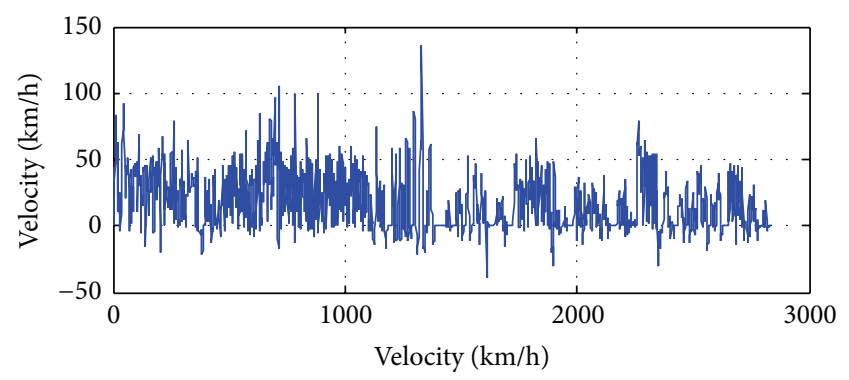

(b)

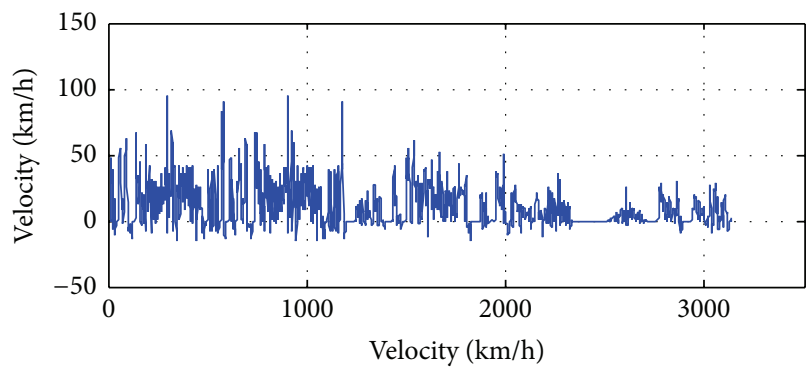

(d)

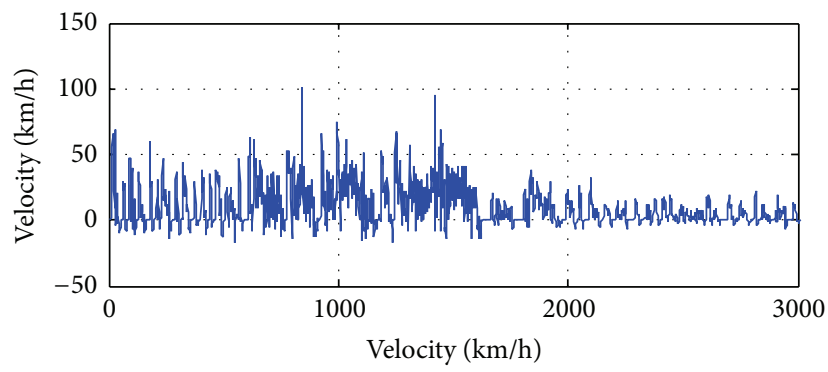

(f)

FIGURE 9: The adopted driving cycles in simulation study. (a), (b) Speed profile and power request, respectively, in cycle 1. (c), (d) Speed profile and power request, respectively, in cycle 2. (e), (f) Speed profile and power request, respectively, in cycle 3.

Critical Condition 3: Low Power Request and High SoC. The vehicle is operated at CD manner when $P_{\text {req }}$ is below $P_{M}$ and battery SoC is higher than $\mathrm{SoC}_{\text {Low }}$.

In control algorithm of bottom layer, power allocation is based on the optimization result from DP. Two thresholds are used to divide the engine power into three parts according to power request. To achieve the minimum of cost function, DP turns engine off when the power request is low. This control policy reduces the energy consumption but increases the start-stop times of engine. Consequently, the power allocation is modified when power request is low. The power allocation control policy in bottom layer is described as

if $P_{\text {req }}(t)>P_{M}$ and $\mathrm{SoC} \geq \mathrm{SoC}_{\mathrm{Low}}$, calculate:

$$
P_{E}(t)= \begin{cases}\chi_{2} & \text { if } P_{\text {req }}(t)>\chi_{2} \\ P_{\text {req }}(t) & \text { if } \chi_{1}<P_{\text {req }}(t) \leq \chi_{2} \\ \chi_{1} & \text { if } P_{\text {req }}(t) \leq \chi_{1}\end{cases}
$$

else if $\mathrm{SoC}<\mathrm{SoC}_{\mathrm{Low}}$, calculate:

$$
\begin{aligned}
& P_{E}(t) \\
& = \begin{cases}\min \left\{P_{E_{-} \text {max }}, P_{\text {req }}(t)\right\} & \text { if } P_{\text {req }}(t)>\chi_{1} \\
\chi_{1} & \text { if } P_{\text {req }}(t) \leq \chi_{1}\end{cases}
\end{aligned}
$$

else

$$
P_{E}(t)=0
$$

where $P_{e_{-} \max }$ is the maximum of engine power and $\chi_{1}$ and $\chi_{2}$ are two thresholds of engine power which are predetermined by DP.

\section{Simulation Results and Discussion}

The presented energy management approach is evaluated by a simulation study in this section. Three driving cycles are used as simulation conditions. The speed profiles and power requests of the adopted driving cycles are shown in Figure 9. 


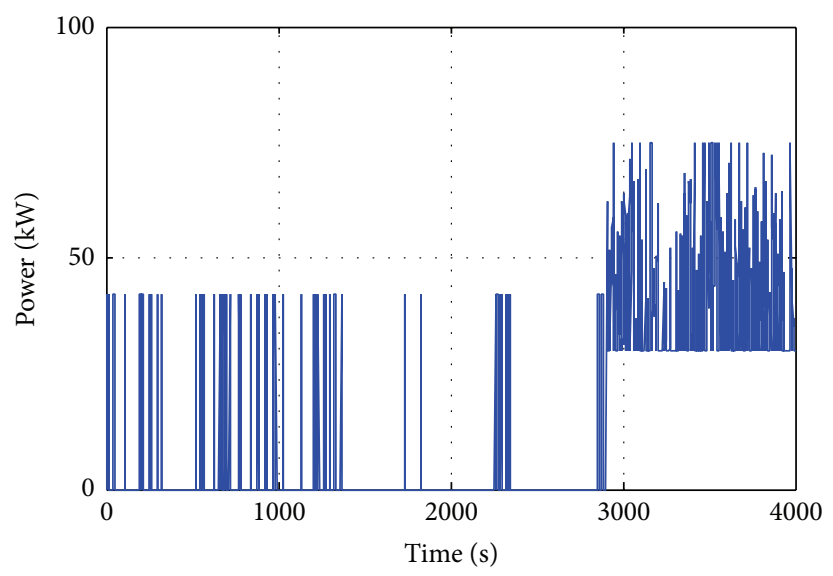

(a)

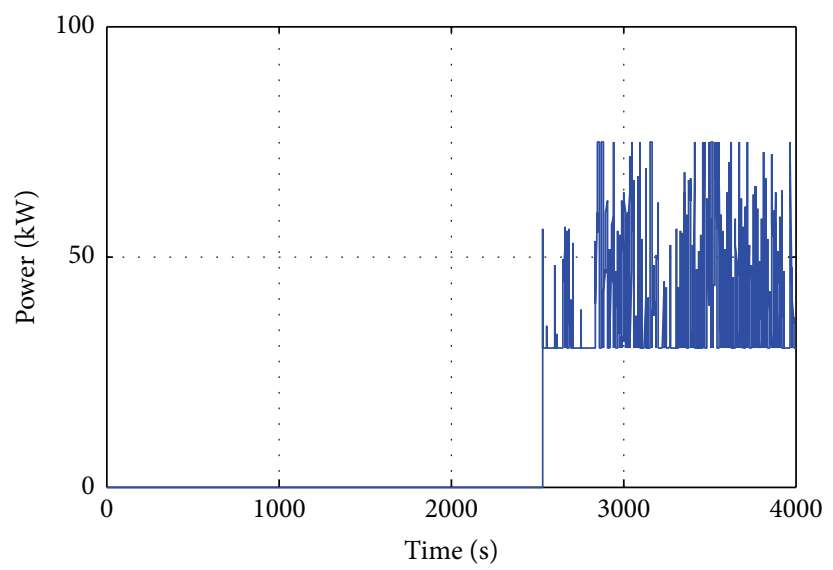

(c)

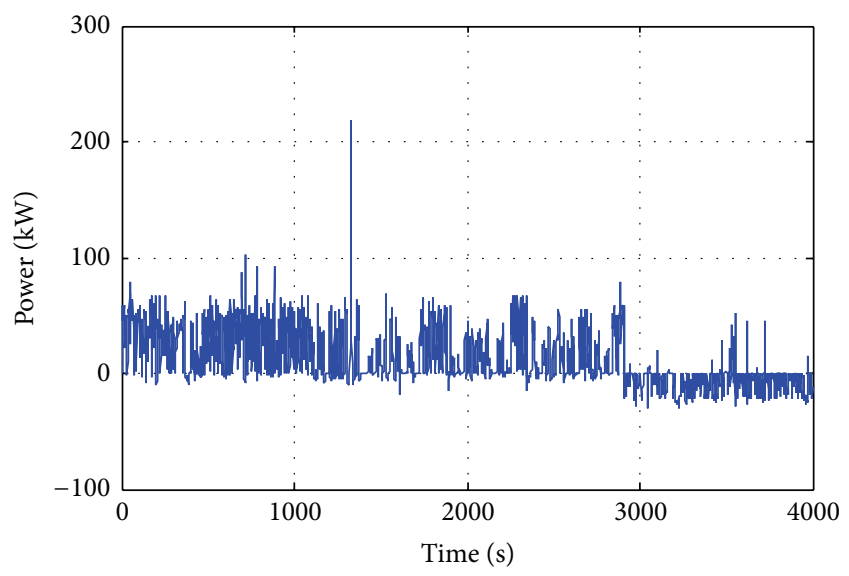

(b)

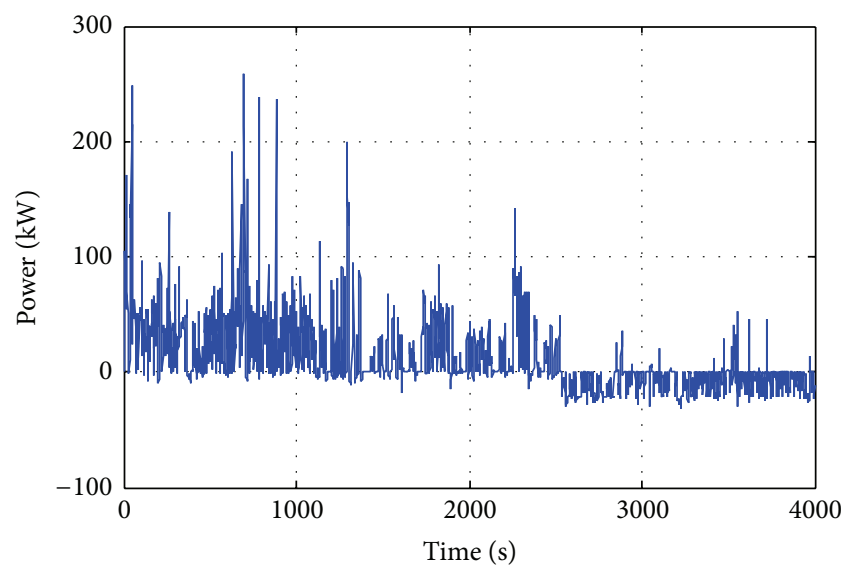

(d)

FIGURE 10: Simulation results of power allocation. (a), (b) Engine power and battery power, respectively, in presented strategy. (c), (d) Engine power and battery power, respectively, in CD-CS strategy.

To better analyze and assess the effectiveness of the presented online energy management approach, the traditional CD-CS strategy is adopted as a benchmark.

The simulation is repeated through the driving cycle until the driving distance exceeds $60 \mathrm{~km}$ to make sure that the CS manner could be reached. Results of power allocation of two strategies in the three driving cycles are given in Figures 1012 , respectively. The length of all-electric range is defined as the summation of driving distance covered by CD manner. The engine power indicates the distribution of CD manner in both strategies. In the presented energy management strategy, the $\mathrm{CD}$ manner and the $\mathrm{CS}^{(P)}$ manner are interlaced while the boundary of CD manner and CS manner is quite clear in CD-CS strategy. This control performance will refrain the battery from the overquick electricity consumption rate when the battery SoC is high. At the last part of simulation, the engine power is enhanced and battery no longer outputs power because the electricity in battery is exhausted and the $\mathrm{CS}^{(B)}$ manner is switched on. From the simulation result of battery power, it is clear that the electricity consumption rate in the presented strategy is lower than that in CD-CS
TABLE 3: The AER results in three cycles.

\begin{tabular}{lccc}
\hline Cycles & $\begin{array}{c}\text { The presented } \\
\text { strategy }\end{array}$ & $\begin{array}{c}\text { CD-CS } \\
\text { strategy }\end{array}$ & Prolongation \\
\hline 1 & $45.76 \mathrm{~km}$ & $44.61 \mathrm{~km}$ & $2.58 \%$ \\
2 & $64.65 \mathrm{~km}$ & $62.85 \mathrm{~km}$ & $2.86 \%$ \\
3 & $65.16 \mathrm{~km}$ & $64.03 \mathrm{~km}$ & $1.76 \%$ \\
\hline
\end{tabular}

strategy at most situations and most of the transient large power discharge situations in CD-CS strategy are eliminated in the presented strategy. The lower battery power can result in a more healthy battery working condition and a probably longer AER. The lengths of AER with two strategies have been recorded in three simulation driving cycles, described in Table 3. Compared to traditional CD-CS strategy, the presented strategy can prolong the ARE by up to $2.86 \%$ at certain driving condition.

To assess the energy saving performance of the presented strategy with uncertain driving distance, the comparison of energy cost between presented strategy and traditional 


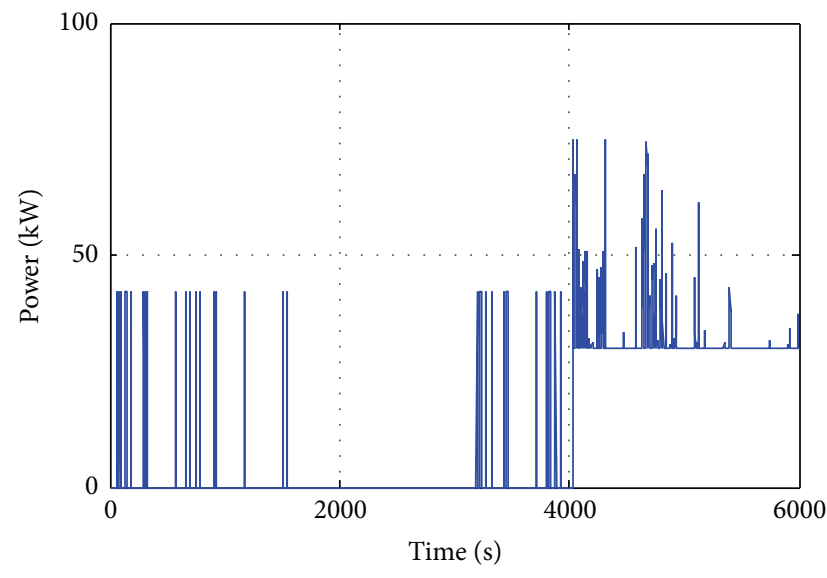

(a)

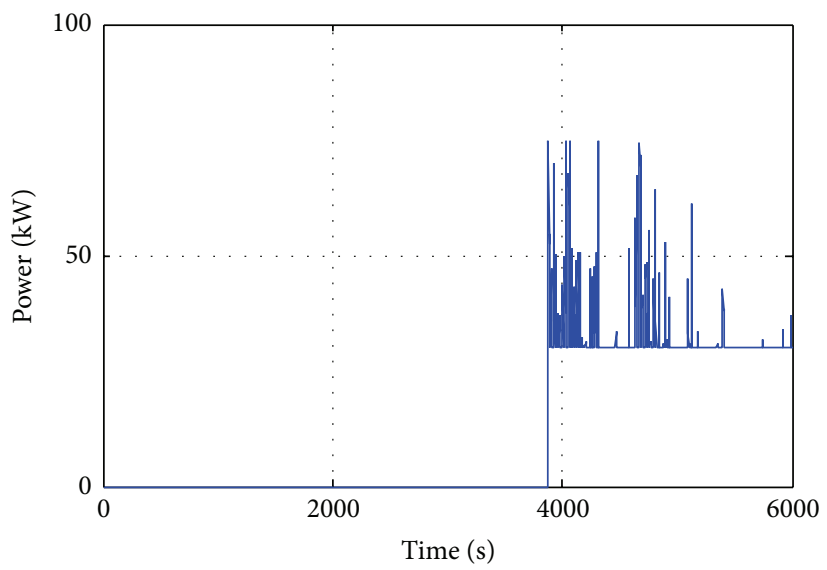

(c)

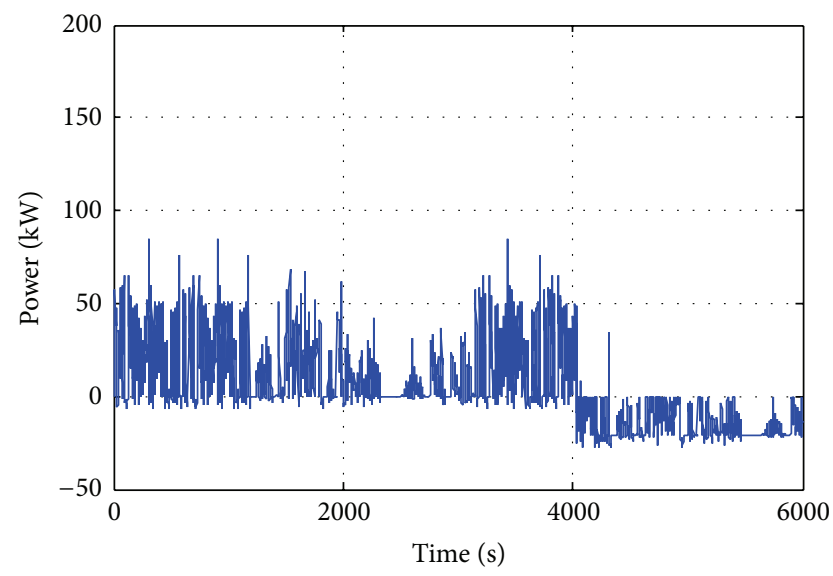

(b)

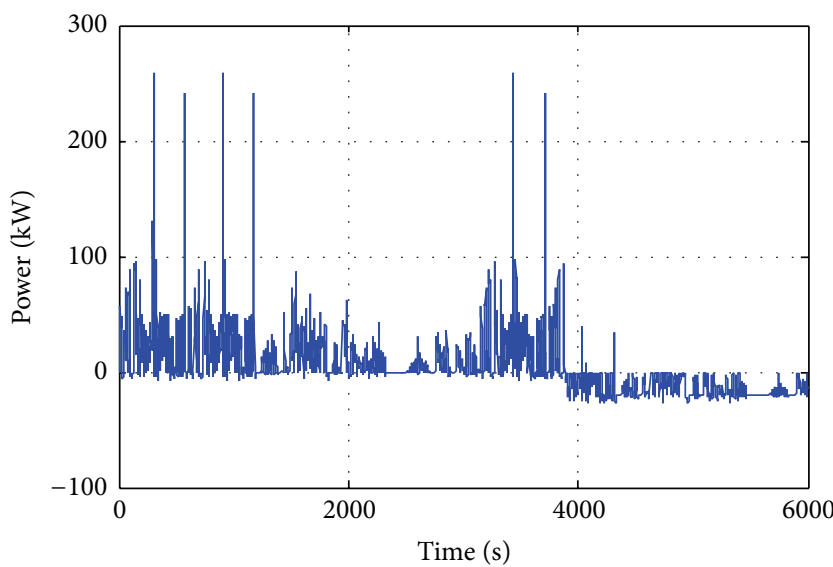

(d)

FIGURE 11: Simulation results of power allocation. (a), (b) Engine power and battery power, respectively, in presented strategy. (c), (d) Engine power and battery power, respectively, in CD-CS strategy.

CD-CS strategy is calculated at $20 \mathrm{~km}, 40 \mathrm{~km}$, and $60 \mathrm{~km}$, respectively. The comparison results in three driving cycles are given in Figure 13. Apparently, the performance of energy saving in the presented strategy is relative to the driving distance. When the trip is quite short, the traditional CD-CS strategy has an obvious advantage. When driving distance is $40 \mathrm{~km}$, energy cost with the presented strategy is lower than that with traditional CD-CS strategy by $5.77 \%$ in driving cycle 1. When the driving distance reaches $60 \mathrm{~km}$, the reduction of energy consumption compared to CD-CS strategy is $4.28 \%, 4.67 \%$, and $4.06 \%$, respectively, in three driving cycles. This result indicates that the presented strategy can achieve a better energy economy when driving distance is quite long, but for a short distance trip, the traditional CD-CS strategy still owns its advantage in terms of energy saving performance.

\section{Conclusions}

A novel DP-based online energy management approach is proposed for the plug-in HEVs in this paper. The presented approach utilizes control policy extracted from optimization results of DP to reduce the energy consumption. Both the power request and the battery SoC are used to control the operation manners for the prolongation of AER. Three driving cycles are employed to evaluate the presented strategy. Simulation results indicate that the AER in the presented approach can be extended by up to $2.86 \%$ compared to CDCS strategy. The comparison result of energy saving performance between the presented strategy and traditional CD-CS strategy is related to the driving distance. For some certain driving conditions, the reduction of energy consumption can be up to $5.77 \%$.

\section{Conflict of Interests}

The authors declare that there is no conflict of interests regarding the publication of this paper.

\section{Acknowledgments}

This work has been supported by the Fundamental Research Funds for the Central Universities (N130403014) and the 


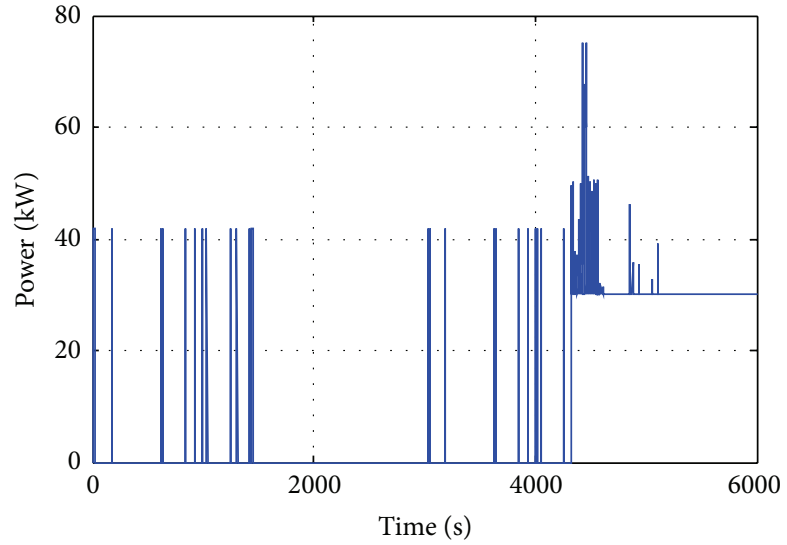

(a)

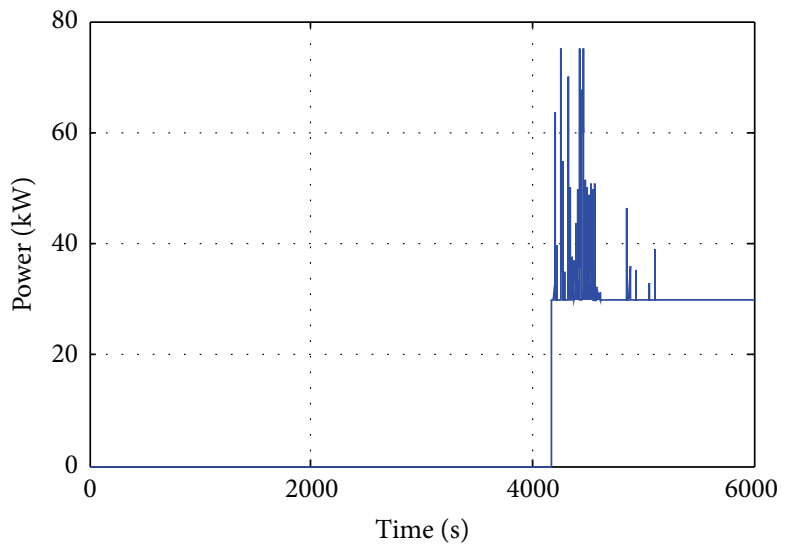

(c)

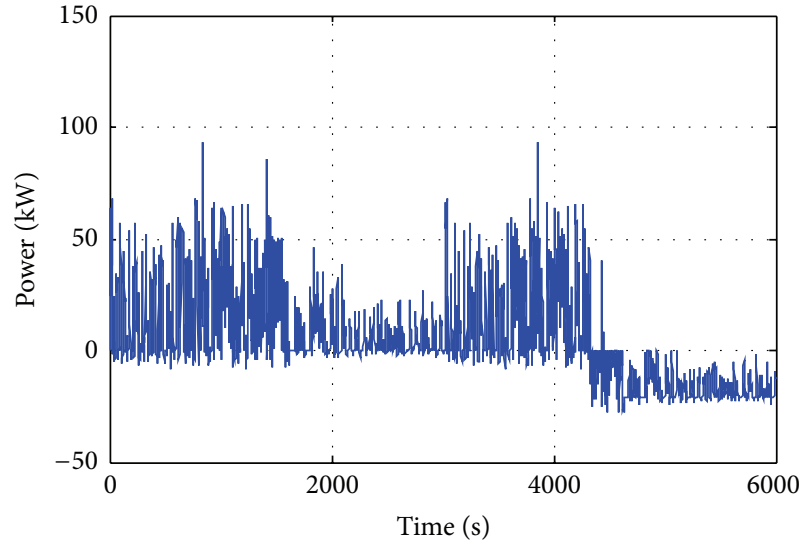

(b)

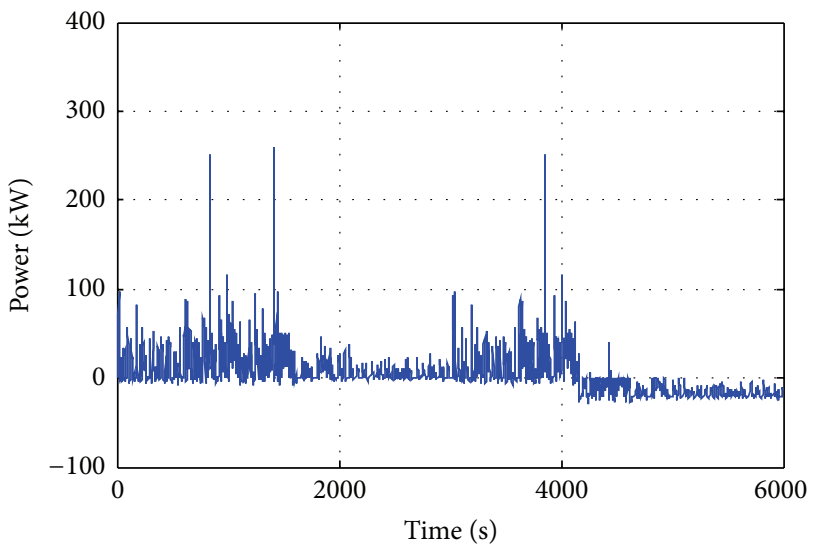

(d)

FIGURE 12: Simulation results of power allocation. (a), (b) Engine power and battery power, respectively, in presented strategy. (c), (d) Engine power and battery power, respectively, in CD-CS strategy.

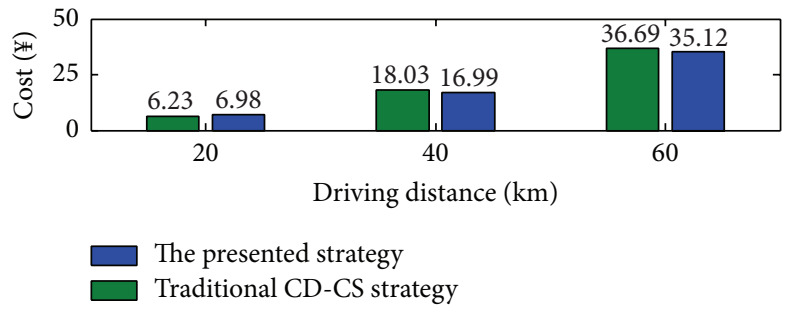

(a)

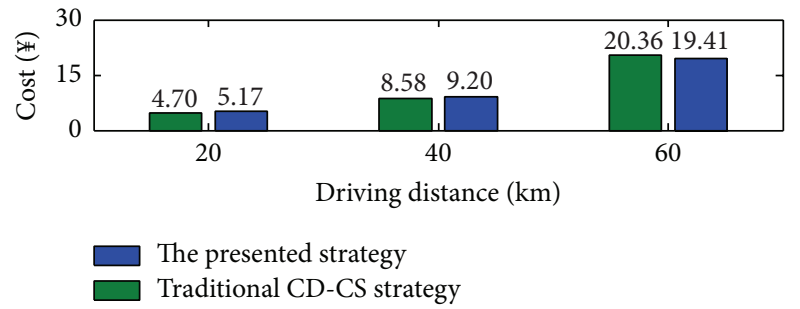

(b)

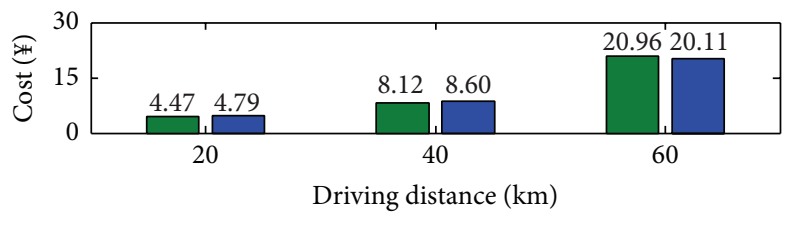

The presented strategy

Traditional CD-CS strategy

(c)

Figure 13: Comparison of energy consumption at different driving distances in three driving cycles. (a) Driving cycle 1; (b) driving cycle 2; (c) driving cycle 3 . 
Open Research Fund of Key Laboratory of Automobile Engineering, Xinhua University (S2jj2012-036).

\section{References}

[1] B. M. Al-Alawi and T. H. Bradley, "Review of hybrid, plug-in hybrid, and electric vehicle market modeling studies," Renewable and Sustainable Energy Reviews, vol. 21, pp. 190-203, 2013.

[2] N. Rotering and M. Ilic, "Optimal charge control of plug-in hybrid electric vehicles in deregulated electricity markets," IEEE Transactions on Power Systems, vol. 26, no. 3, pp. 1021-1029, 2011.

[3] A. A. Malikopoulos, "Supervisory power management control algorithms for hybrid electric vehicles: a survey," IEEE Transactions on Intelligent Transportation Systems, vol. 15, no. 5, pp. 1869-1885, 2014.

[4] S. G. Wirasingha and A. Emadi, "Classification and review of control strategies for plug-in hybrid electric vehicles," IEEE Transactions on Vehicular Technology, vol. 60, no. 1, pp. 111-122, 2011.

[5] H. W. He, R. Xiong, K. Zhao, and Z. Liu, "Energy management strategy research on a hybrid power system by hardware-inloop experiments," Applied Energy, vol. 112, pp. 1311-1317, 2013.

[6] Y.-H. Hung and C.-H. Wu, "An integrated optimization approach for a hybrid energy system in electric vehicles," Applied Energy, vol. 98, pp. 479-490, 2012.

[7] X. Hu, L. Johannesson, N. Murgovski, and B. Egardt, "Longevity-conscious dimensioning and power management of the hybrid energy storage system in a fuel cell hybrid electric bus," Applied Energy, vol. 137, pp. 913-924, 2015.

[8] Z. Chen, R. Xiong, K. Wang, and B. Jiao, "Optimal energy management strategy of a plug-in hybrid electric vehicle based on a particle swarm optimization algorithm," Energies, vol. 8, no. 5, pp. 3661-3678, 2015.

[9] X. Hu, N. Murgovski, L. M. Johannesson, and B. Egardt, "Optimal dimensioning and power management of a fuel cell/battery hybrid bus via convex programming," IEEE/ASME Transactions on Mechatronics, vol. 20, no. 1, pp. 457-468, 2014.

[10] D. F. Opila, X. Wang, R. McGee, R. B. Gillespie, J. A. Cook, and J. W. Grizzle, "An energy management controller to optimally trade off fuel economy and drivability for hybrid vehicles," IEEE Transactions on Control Systems Technology, vol. 20, no. 6, pp. 1490-1505, 2012.

[11] Y. del Valle, G. K. Venayagamoorthy, S. Mohagheghi, J.-C. Hernandez, and R. G. Harley, "Particle swarm optimization: basic concepts, variants and applications in power systems," IEEE Transactions on Evolutionary Computation, vol. 12, no. 2, pp. 171-195, 2008.

[12] S. Zhang and R. Xiong, "Adaptive energy management of a plugin hybrid electric vehicle based on driving pattern recognition and dynamic programming," Applied Energy, vol. 155, pp. 6878, 2015.

[13] L. V. Pérez and E. A. Pilotta, "Optimal power split in a hybrid electric vehicle using direct transcription of an optimal control problem," Mathematics and Computers in Simulation, vol. 79, no. 6, pp. 1959-1970, 2009.

[14] D. Kum, H. Peng, and N. K. Bucknor, "Supervisory control of parallel hybrid electric vehicles for fuel and emission reduction," Journal of Dynamic Systems, Measurement and Control, vol. 133, no. 6, Article ID 061010, 10 pages, 2011.

[15] L. Wang, Y. Zhang, C. Yin, H. Zhang, and C. Wang, "Hardwarein-the-loop simulation for the design and verification of the control system of a series-parallel hybrid electric city-bus," Simulation Modelling Practice and Theory, vol. 25, pp. 148-162, 2012.

[16] S. J. Moura, H. K. Fathy, D. S. Callaway, and J. L. Stein, "A stochastic optimal control approach for power management in plug-in hybrid electric vehicles," IEEE Transactions on Control Systems Technology, vol. 19, no. 3, pp. 545-555, 2011.

[17] E. D. Tate, J. W. Grizzle, and H. Peng, "SP-SDP for fuel consumption and tailpipe emissions minimization in an EVT hybrid," IEEE Transactions on Control Systems Technology, vol. 18, no. 3, pp. 673-687, 2010.

[18] L. Johannesson, M. Åsbogård, and B. Egardt, "Assessing the potential of predictive control for hybrid vehicle powertrains using stochastic dynamic programming," IEEE Transactions on Intelligent Transportation Systems, vol. 8, no. 1, pp. 71-83, 2007.

[19] W. Li, T.-K. Lee, Z. S. Filipi, X. Meng, and C.-N. Zhang, "Development of electric machine duty cycles for parallel hybrid electric Beijing city bus based on Markov chain," International Journal of Vehicle Design, vol. 58, no. 2-4, pp. 348-366, 2012.

[20] L. Serrao, S. Onori, and G. Rizzoni, "A comparative analysis of energy management strategies for hybrid electric vehicles," Journal of Dynamic Systems, Measurement and Control, vol. 133, no. 3, Article ID 031012, 9 pages, 2011.

[21] X. Hu, N. Murgovski, L. Johannesson, and B. Egardt, "Energy efficiency analysis of a series plug-in hybrid electric bus with different energy management strategies and battery sizes," Applied Energy, vol. 111, pp. 1001-1009, 2013. 


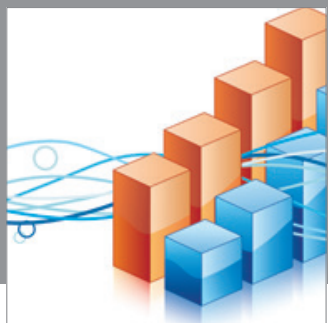

Advances in

Operations Research

mansans

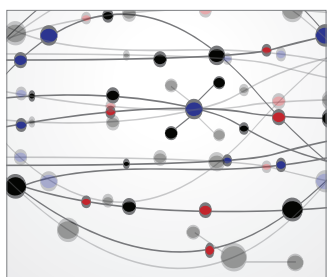

The Scientific World Journal
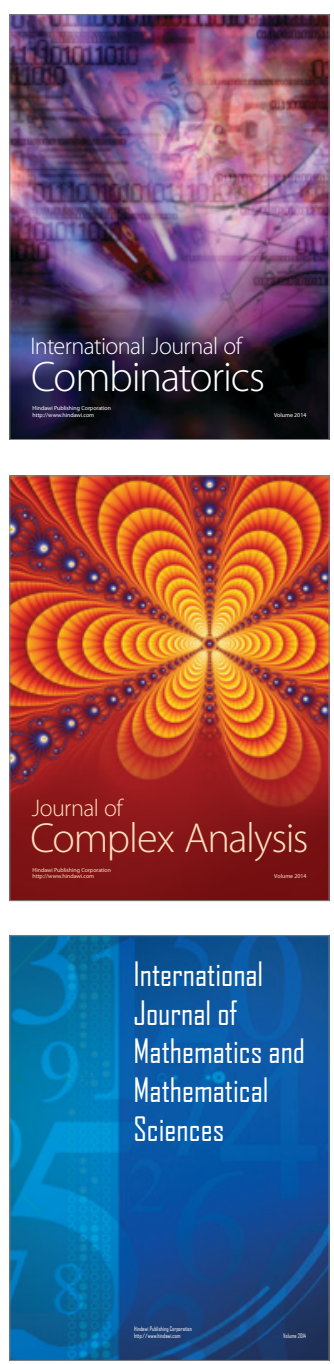
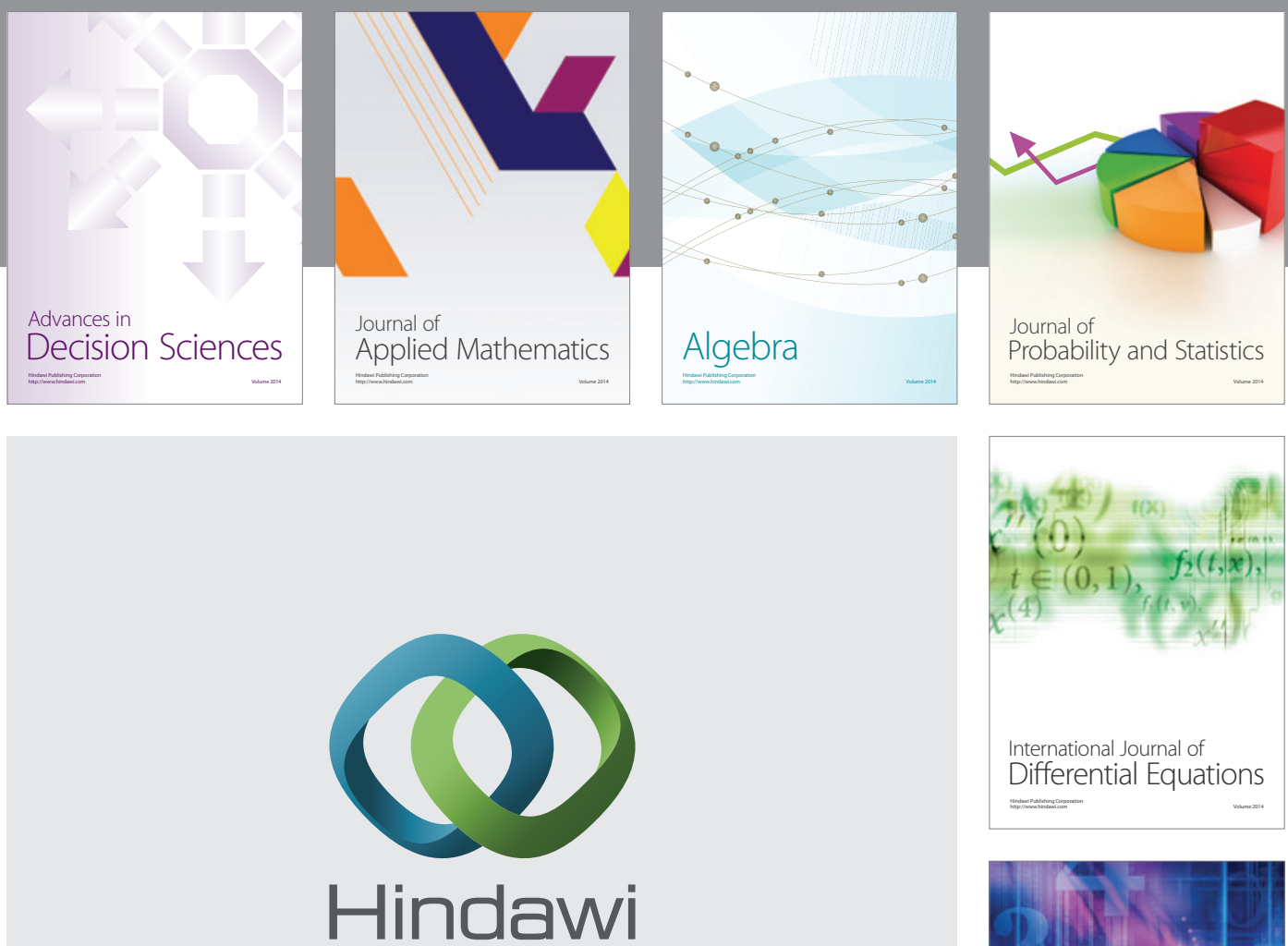

Submit your manuscripts at http://www.hindawi.com
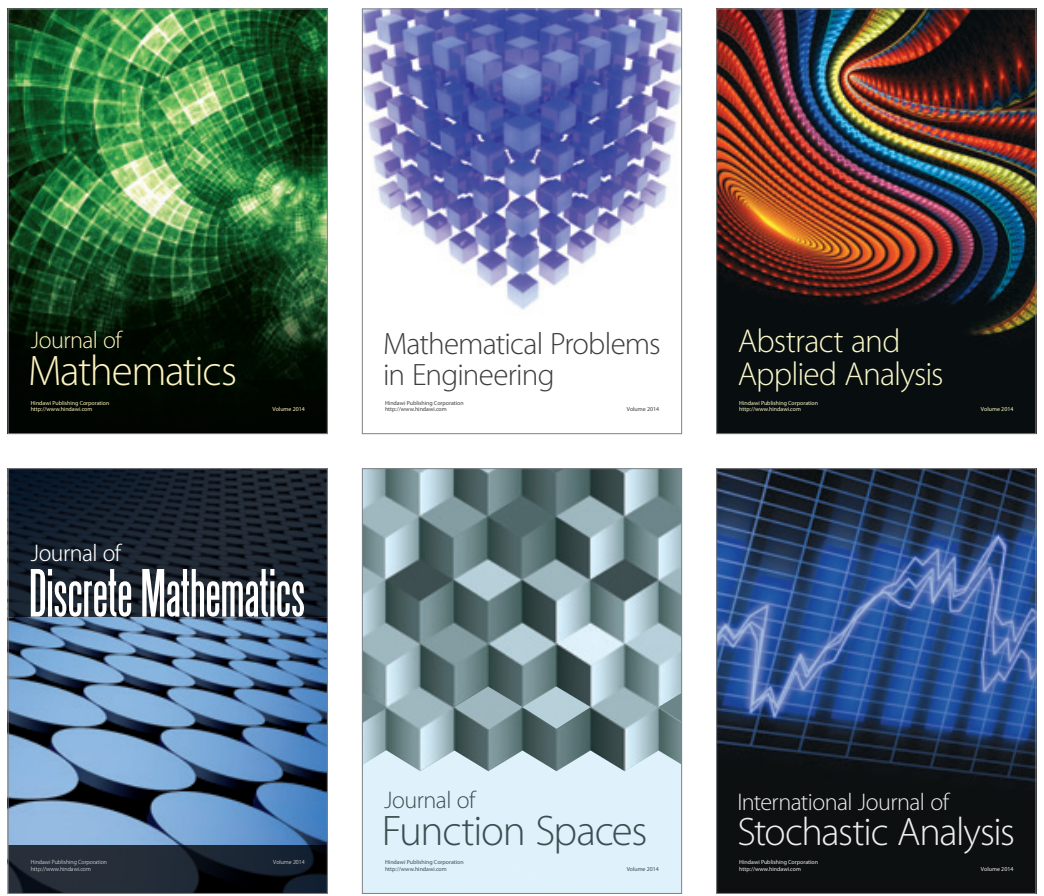

Journal of

Function Spaces

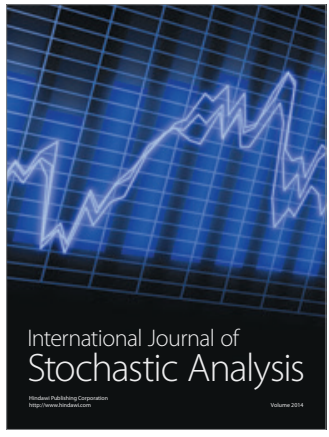

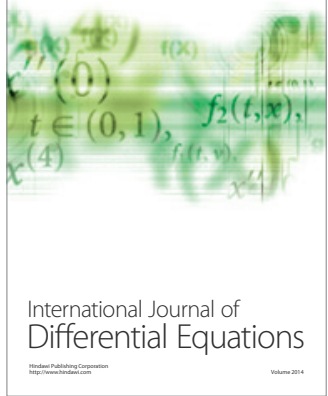
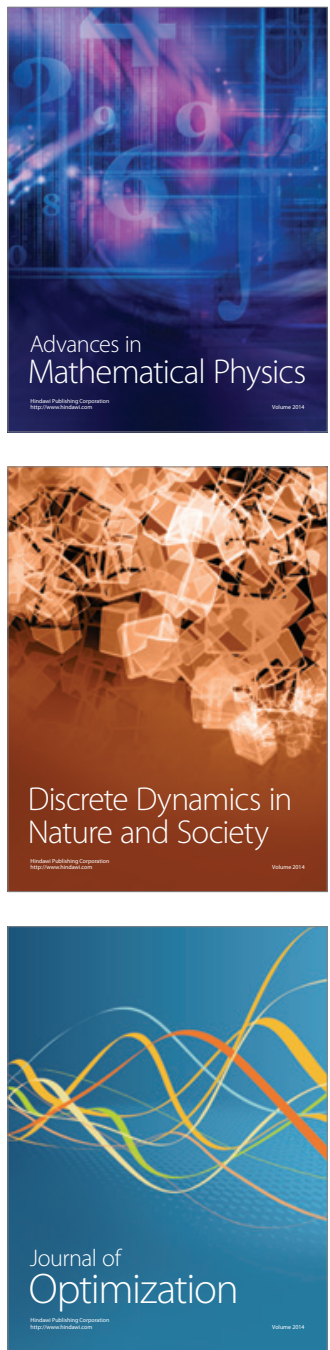